International Journal of Pure and Applied Mathematics

Volume 95 No. 1 2014, 13-22

ISSN: 1311-8080 (printed version); ISSN: 1314-3395 (on-line version)

url: http://www.ijpam.eu

doi: http://dx.doi.org/10.12732/ijpam.v95i1.2

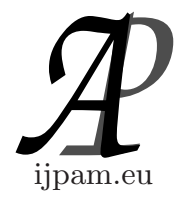

\title{
FIXED POINT AND APPROXIMATELY COMPOSITE FUNCTIONAL EQUATIONS IN NON-ARCHIMEDEAN NORMED SPACES
}

Mohammad Hadi Eghtesadifard ${ }^{1}$, Dong Yun Shin ${ }^{2}$, Hassan Azadi Kenary ${ }^{3}$, Najmeh Sahami ${ }^{4}$

${ }^{1}$ Instructor of Fars Education Department

Shiraz, IRAN

${ }^{2}$ Department of Mathematics

College of Natural Science

University of Seoul

KOREA

${ }^{3,4}$ Department of Mathematics

Beyza Branch

Islamic Azad University

Beyza, IRAN

\begin{abstract}
In this paper, we investigate the generalized Hyers-Ulam-Rassias(or Hyers-Ulam) stability of a composite additive functional equation in non-Archimedean normed spaces.

The concept of Hyers-Ulam-Rassias or Hyers-Ulam stability originated from the Th.M. Rassias stability theorem that appeared in his paper: On the stability of the linear mapping in Banach spaces, Proc. Amer. Math. Soc., 72 (1978), 297-300.
\end{abstract}

AMS Subject Classification: 39B22, 39B52, 39B22, 39B82, 46S10 Key Words: Hyers-Ulam stability, stability, non-Archimedean normed spaces

Received: April 29, 2013

(c) 2014 Academic Publications, Ltd.

$\S$ Correspondence author url: www.acadpubl.eu 


\section{Introduction and Preliminaries}

A classical question in the theory of functional equations is the following: When is it true that a function which approximately satisfies a functional equation $D$ must be close to an exact solution of $D$ ?

If the problem accepts a solution, we say that the equation $D$ is stable. The first stability problem concerning group homomorphisms was raised by Ulam [26] in 1940.

We are given a group $G$ and a metric group $G^{\prime}$ with metric $d(.,$.$) . Given \varepsilon>$ 0 , dose there exist a $\delta>0$ such that if $f: G \rightarrow G^{\prime}$ satisfies $d(f(x y), f(x) f(y))<$ $\delta$, for all $x, y \in G$, then a homomorphism $h: G \rightarrow G$; exists with $d(f(x), h(x))<$ $\varepsilon$ for all $x \in G$ ?

Ulam's problem was partially solved by Hyers [10] in 1941.

Let $E_{1}$ be a normed space, $E_{2}$ a Banach space and suppose that the mapping $f: E_{1} \rightarrow E_{2}$ satisfies the inequality

$$
\|f(x+y)-f(x)-f(y)\| \leq \varepsilon
$$

for all $x, y \in E_{1}$, where $\varepsilon>0$ is a constant. Then the limit

$$
T(x)=\lim _{n \rightarrow \infty} 2^{-n} f\left(2^{n} x\right)
$$

exists for each $x \in E_{1}$ and $T$ is the unique additive mapping satisfying

$$
\|T(x)-f(x)\| \leq \varepsilon
$$

for all $x \in E_{1}$. Also, if for each $x$ the function $t \rightarrow f(t x)$ from $\mathbb{R}$ to $E_{2}$ is continuous on $\mathbb{R}$, then $T$ is linear. If $f$ is continuous at a single point of $E_{1}$, then $T$ is continuous everywhere in $E_{1}$. Moreover (1.1) is sharp.

In 1978, Th. M. Rassias [18] formulated and proved the following theorem, which implies Hyers's Theorem as a special case. Suppose that $E$ and $F$ are real normed spaces with $F$ a complete normed space, $f: E \rightarrow F$ is a mapping such that for each fixed $x \in E$ the mapping $t \rightarrow f(t x)$ is continuous on $R$, and let there exist $\varepsilon \geq 0$ and $p \in[0,1)$ such that for all $x, y \in E$

$$
\|f(x+y)-f(x)-f(y)\| \leq \varepsilon\left(\|x\|^{p}+\|y\|^{p}\right)
$$

Then there exists a unique linear mapping $T: E \rightarrow F$ such that such that for all $x \in E$

$$
\|f(x)-T(x)\| \leq \frac{\varepsilon\|x\|^{p}}{1-2^{p-1}}
$$


The case of the existence of a unique additive mapping had been obtained by T. Aoki [2], as it is recently noticed by Lech Maligranda. However, Aoki [2] had claimed the existence of a unique linear mapping, that is not true because he did not allow the mapping $f$ to satisfy some continuity assumption. Th.M. Rassias [18], who independently introduced the unbounded Cauchy difference was the first to prove that there exists a unique linear mapping $\mathrm{T}$ satisfying

$$
\|f(x)-T(x)\| \leq \frac{\epsilon\|x\|^{p}}{1-2^{p-1}} \quad x \in E
$$

In 1990, Th.M. Rassias [19] during the 27th International Symposium on Functional Equations asked the question whether such a theorem can also be proved for $p \geq 1$. In 1991, Z. Gajda [8] following the same approach as in Th. M. Rassias [24], gave an affirmative solution to this question for $p>1$. It was proved by Z. Gajda [8], as well as by Th. M. Rassias and P. Šemrl [20] that one can not prove a Th. M. Rassias type theorem when $p=1$. In 1994, P. Găvruta [9] provided a further generalization of Th. M. Rassias theorem in which he replaced the bound $\varepsilon\left(\|x\|^{p}+\|\| y \|^{p}\right)$ by a general control function $\psi(x, y)$ for the existence of a unique linear mapping.

The functional equation $f(x+y)+f(x-y)=2 f(x)+2 f(y)$ is called the quadratic functional equation. In particular, every solution of the quadratic functional equation is said to be a quadratic mapping. A generalized HyersUlam stability problem for the quadratic functional equation was proved by Skof [25] for mappings $f: X \rightarrow Y$, where $X$ is a normed space and $Y$ is a Banach space. Cholewa [6] noticed that the theorem of Skof is still true if the relevant domain $\mathrm{X}$ is replaced by an Abelian group. In [7], Czerwik proved the generalized Hyers-Ulam stability of the quadratic functional equation.

During the last decades several stability problems of functional equations have been investigated by a number of mathematicians( [1]-[5], [11]-[24]).

Definition 1.1. By a non-Archimedean field we mean a field $\mathbb{K}$ equipped with a function(valuation) $||:. \mathbb{K} \rightarrow[0, \infty)$ such that for all $r, s \in \mathbb{K}$, the following conditions hold:

(i) $|r|=0$ if and only if $r=0$;

(ii) $|r s|=|r||s|$;

(iii) $|r+s| \leq \max \{|r|,|s|\}$.

Definition 1.2. Let $X$ be a vector space over a scalar field $\mathbb{K}$ with a non-Archimedean non-trivial valuation |.| . A function $\|\|:. X \rightarrow \mathbb{R}$ is a nonArchimedean norm (valuation) if it satisfies the following conditions: 
(i) $\|x\|=0$ if and only if $x=0$;

(ii) $\|r x\|=|r|\|x\| \quad(r \in \mathbb{K}, x \in X)$;

(iii) The strong triangle inequality( ultrametric); namely

$$
\|x+y\| \leq \max \{\|x\|,\|y\|\} . \quad x, y \in X
$$

Then $(X,\|\|$.$) is called a non-Archimedean space.$

Due to the fact that

$$
\left\|x_{n}-x_{m}\right\| \leq \max \left\{\left\|x_{j+1}-x_{j}\right\|: m \leq j \leq n-1\right\} \quad(n>m)
$$

Definition 1.3. A sequence $\left\{x_{n}\right\}$ is Cauchy if and only if $\left\{x_{n+1}-x_{n}\right\}$ converges to zero in a non-Archimedean space. By a complete non-Archimedean space we mean one in which every Cauchy sequence is convergent.

Definition 1.4. Let $X$ be a set. A function $d: X \times X \rightarrow[0, \infty]$ is called a generalized metric on $X$ if $d$ satisfies the following conditions:

(a) $d(x, y)=0$ if and only if $x=y$ for all $x, y \in X$;

(b) $d(x, y)=d(y, x)$ for all $x, y \in X$;

(c) $d(x, z) \leq d(x, y)+d(y, z)$ for all $x, y, z \in X$.

Theorem 1.1. Let $(X, d)$ be a complete generalized metric space and $J: X \rightarrow X$ be a strictly contractive mapping with Lipschitz constant $L<1$. Then, for all $x \in X$, either $d\left(J^{n} x, J^{n+1} x\right)=\infty$ for all nonnegative integers $n$ or there exists a positive integer $n_{0}$ such that:

(a) $d\left(J^{n} x, J^{n+1} x\right)<\infty$ for all $n_{0} \geq n_{0}$;

(b) the sequence $\left\{J^{n} x\right\}$ converges to a fixed point $y^{*}$ of $J$;

(c) $y^{*}$ is the unique fixed point of $J$ in the set $Y=\left\{y \in X: d\left(J^{n_{0}} x, y\right)<\right.$ $\infty\}$

(d) $d\left(y, y^{*}\right) \leq \frac{1}{1-L} d(y, J y)$ for all $y \in Y$.

In this paper, we prove the generalized Hyers-Ulam stability of the following functional equation

$$
f(f(x)-f(y))=f(x+y)+f(x-y)-f(x)-f(y)
$$

in non-Archimedean normed spaces. In the rest of the paper let $|2| \neq 1$. 


\section{Non-Archimedean Stability of Eq. (1.3): A Fixed Point Method}

Throughout this section, using the fixed point method we prove the generalized Hyers-Ulam stability of the composite functional equation (1.3) in nonArchimedean spaces.

Theorem 2.1. Let $X$ is a non-Archimedean normed space and that $Y$ be a complete non-Archimedean normed space. Assume $\zeta: X^{2} \rightarrow[0, \infty)$ be a function such that there exists an $L<1$ with

$$
\zeta(2 x, 2 y) \leq|2| L \zeta(x, y)
$$

for all $x, y \in X$. Let $f: X \rightarrow Y$ be a mapping satisfying

$$
\|f(f(x)-f(y))-f(x+y)-f(x-y)+f(x)+f(y)\| \leq \zeta(x, y)
$$

for all $x, y \in X$. Then there is a unique additive mapping $A: X \rightarrow Y$ such that

$$
\|f(x)-A(x)\| \leq \frac{\zeta(x, x)}{|2|-|2| L}
$$

Proof. Putting $y=x$ in (2.2), we have

$$
\left\|\frac{f(2 x)}{2}-f(x)\right\| \leq \frac{1}{|2|} \zeta(x, x)
$$

for all $x \in X$. Consider the set $S:=\{g: X \rightarrow Y\}$ and the generalized metric $d$ in $S$ defined by $d(f, g)=\inf _{\mu \in(0,+\infty)}\{\|g(x)-h(x)\| \leq \mu \zeta(x, x), \forall x \in X\}$, where $\inf \emptyset=+\infty$. It is easy to show that $(S, d)$ is complete (see [12], Lemma 2.1).

Now, we consider a linear mapping $J:(S, d) \rightarrow(S, d)$ such that $J h(x):=$ $\frac{1}{2} h(2 x)$ for all $x \in X$. Let $g, h \in S$ be such that $d(g, h)=\epsilon$. Then $\| g(x)-$ $h(x) \| \leq \epsilon \zeta(x, x)$ for all $x \in X$ and so

$$
\|J g(x)-J h(x)\|=\left\|\frac{g(2 x)}{2}-\frac{h(2 x)}{2}\right\| \leq \frac{1}{|2|} \epsilon \zeta(2 x, 2 x) \leq \frac{1}{|2|} \epsilon|2| L \zeta(x, x)
$$

for all $x \in X$. Thus $d(g, h)=\epsilon$ implies that $d(J g, J h) \leq L \epsilon$. This means that $d(J g, J h) \leq L d(g, h)$ for all $g, h \in S$. It follows from (2.4) that $d(f, J f) \leq$ $\frac{1}{2}<+\infty$. By Theorem 1.1, there exists a mapping $A: X \rightarrow Y$ satisfying the following:

(1) $A$ is a fixed point of $J$, that is,

$$
A(2 x)=2 A(x)
$$


for all $x \in X$. The mapping $A$ is a unique fixed point of $J$ in the set $\Omega=\{h \in$ $S: d(g, h)<\infty\}$. This implies that $A$ is a unique mapping satisfying (2.5) such that there exists $\mu \in(0, \infty)$ satisfying $\|f(x)-A(x)\| \leq \mu \zeta(x, x)$ for all $x \in X$.

(2) $d\left(J^{n} f, A\right) \rightarrow 0$ as $n \rightarrow \infty$. This implies the equality $\lim _{n \rightarrow \infty} \frac{f\left(2^{n} x\right)}{2^{n}}=$ $A(x)$ for all $x \in X$.

(3) $d(f, A) \leq \frac{d(f, J f)}{1-L}$ with $f \in \Omega$, which implies the inequality $d(f, A) \leq$ $\frac{1}{|2|-|2| L}$. This implies that the inequality (2.3) holds. By (2.1) and (2.2), we obtain

$$
\begin{aligned}
& \|A(A(x)-A(y))-A(x+y)-A(x-y)+A(x)+A(y)\| \\
& \lim _{\mathrm{n} \rightarrow \infty}\left\|\frac{f\left(f\left(2^{\mathrm{n}} x\right)-f\left(2^{\mathrm{n}} y\right)\right)}{2^{\mathrm{n}}}-\frac{f\left(2^{\mathrm{n}} x+2^{\mathrm{n}} y\right)}{2^{\mathrm{n}}}-\frac{f\left(2^{\mathrm{n}} x-2^{\mathrm{n}} y\right)}{2^{\mathrm{n}}}+\frac{f\left(2^{\mathrm{n}} x\right)}{2^{\mathrm{n}}}+\frac{f\left(2^{\mathrm{n}} y\right)}{2^{\mathrm{n}}}\right\| \\
& \leq \lim _{\mathrm{n} \rightarrow \infty} \frac{1}{|2|^{\mathrm{n}}} \zeta\left(2^{\mathrm{n}} x, 2^{\mathrm{n}} y\right) \leq \lim _{\mathrm{n} \rightarrow \infty} \frac{1}{|2|^{\mathrm{n}}} \cdot L^{\mathrm{n}} \cdot|2|^{\mathrm{n}} \zeta(x, y)=0
\end{aligned}
$$

for all $x, y \in X$ and $n \in \mathbb{N}$. So $\| A(A(x)-A(y))-A(x+y)-A(x-y)+A(x)+$ $A(y) \|=0$, for all $x, y \in X$. Thus the mapping $A: X \rightarrow Y$ satisfying in (1.3).

On the other hand

$$
2 A\left(\frac{x}{2}\right)-A(x)=\lim _{n \rightarrow \infty} \frac{f\left(2^{n-1} x\right)}{2^{n-1}}-\lim _{n \rightarrow \infty} \frac{f\left(2^{n} x\right)}{2^{n}}=0 .
$$

So, $A$ is an additive mapping, as desired. This completes the proof.

Corollary 2.1. Let $\theta \geq 0$ and $r$ be a real number with $0<r<1$. Let $f: X \rightarrow Y$ be a mapping satisfying

$$
\|f(f(x)-f(y))-f(x+y)-f(x-y)+f(x)+f(y)\| \leq \theta\left(\|x\|^{r}+\|y\|^{r}\right)
$$

for all $x, y \in X$. Then the limit $A(x)=\lim _{n \rightarrow \infty} \frac{f\left(2^{\mathrm{n}} x\right)}{2^{\mathrm{n}}}$ exists for all $x \in X$ and $A: X \rightarrow Y$ is a unique additive mapping such that

$$
\|f(x)-A(x)\| \leq \frac{2 \theta\|x\|^{r}}{|2|-|2|^{r+1}}
$$

for all $x \in X$.

Proof. The proof follows from Theorem 2.1 by taking $\zeta(x, y)=\theta\left(\|x\|^{r}+\right.$ $\|y\|^{r}$ ) for all $x, y \in X$. In fact, if we choose $L=|2|^{r}$, then we get the desired result. 
Theorem 2.2. Let $X$ is a non-Archimedean normed space and that $Y$ be a complete non-Archimedean normed space. Assume $\zeta: X^{2} \rightarrow[0, \infty)$ be a function such that there exists an $L<1$ with

$$
\zeta\left(\frac{x}{2}, \frac{y}{2}\right) \leq \frac{L}{|2|} \zeta(x, y)
$$

for all $x, y \in X$. Let $f: X \rightarrow Y$ be a mapping satisfying (2.2). Then the limit $A(x)=\lim _{n \rightarrow \infty} 2^{n} f\left(\frac{x}{2^{n}}\right)$ exists for all $x \in X$ and defines a unique additive mapping $A: X \rightarrow Y$ such that

$$
\|f(x)-A(x)\| \leq \frac{L \zeta(x, x)}{|2|-|2| L}
$$

Proof. Substituting $y=x$ in (2.2) and then replacing $x$ by $\frac{x}{2}$, we get

$$
\left\|f(x)-2 f\left(\frac{x}{2}\right)\right\| \leq \zeta\left(\frac{x}{2}, \frac{x}{2}\right)
$$

for all $x \in X$. Let $(S, d)$ be the generalized metric space defined in the proof of Theorem 2.1. Consider a linear mapping $J:(S, d) \rightarrow(S, d)$ such that $J h(x):=2 h\left(\frac{x}{2}\right)$ for all $x \in X$. Let $g, h \in S$ be such that $d(g, h)=\epsilon$. Then $\|g(x)-h(x)\| \leq \epsilon \zeta(x, x)$ for all $x \in X$ and so

$$
\|J g(x)-J h(x)\|=\left\|2 g\left(\frac{x}{2}\right)-2 h\left(\frac{x}{2}\right)\right\| \leq|2| \epsilon \zeta\left(\frac{x}{2}, \frac{x}{2}\right) \leq|2| \epsilon \cdot \frac{L}{|2|} \zeta(x, x)
$$

for all $x \in X$. Thus $d(g, h)=\epsilon$ implies that $d(J g, J h) \leq L \epsilon$. This means that $d(J g, J h) \leq L d(g, h)$ for all $g, h \in S$. It follows from $(2.8)$ that $d(f, J f) \leq \frac{L}{22}$.

By Theorem 1.1, there exists a mapping $A: X \rightarrow Y$ satisfying the following:

(1) $A$ is a fixed point of $J$, that is,

$$
A\left(\frac{x}{2}\right)=\frac{1}{2} A(x)
$$

for all $x \in X$. The mapping $A$ is a unique fixed point of $J$ in the set $\Omega=\{h \in$ $S: d(g, h)<\infty\}$. This implies that $A$ is a unique mapping satisfying (2.9) such that there exists $\mu \in(0, \infty)$ satisfying $\|f(x)-A(x)\| \leq \mu \zeta(x, x)$ for all $x \in X$.

(2) $d\left(J^{n} f, A\right) \rightarrow 0$ as $n \rightarrow \infty$. This implies the equality $\lim _{n \rightarrow \infty} 2^{n} f\left(\frac{x}{2^{n}}\right)=$ $A(x)$ for all $x \in X$.

(3) $d(f, A) \leq \frac{d(f, J f)}{1-L}$ with $f \in \Omega$, which implies the inequality $d(f, A) \leq$ $\frac{L}{|2|-|2| L}$. The rest of the proof is similar to the proof of Theorem 2.1. 
Corollary 2.2. Let $\theta \geq 0$ and $r$ be a real number with $r>1$. Let $f$ : $X \rightarrow Y$ be a mapping satisfying (2.6). Then the limit $A(x)=\lim _{n \rightarrow \infty} 8^{n} f\left(\frac{x}{2^{n}}\right)$ exists for all $x \in X$ and $A: X \rightarrow Y$ is a unique additive mapping such that

$$
\|f(x)-A(x)\| \leq \frac{2|2|^{r-1} \theta\|x\|^{r}}{|2|-|2|^{r}}
$$

for all $x \in X$.

Proof. The proof follows from Theorem 2.2 by taking $\zeta(x, y)=\theta\left(\|x\|^{r}+\right.$ $\|y\|^{r}$ ) for all $x, y \in X$. In fact, if we choose $L=|2|^{r-1}$, then we get the desired result.

\section{Acknowledgments}

D.Y. Shin was supported by Basic Science Research Program through the National Research Foundation of Korea funded by the Ministry of Education, Science and Technology (NRF-2010-0021792).

\section{References}

[1] L. M. Arriola and W. A. Beyer, Stability of the Cauchy functional equation over p-adic fields, Real Anal. Exchange 31 (2005/06), no. 1, 125-132.

[2] T. Aoki, On the stability of the linear transformationin Banach spaces, J. Math. Soc. Japan, 2 (1950), 64-66.

[3] H. Azadi Kenary, Hyres-Rassias Stability of The Pexiderial Functional Equation, Italian Journal of Pure and Applied Mathematics (in press).

[4] H. Azadi Kenary, The Probabilistic Stability of a Pexiderial Functional Equation in Random Normed Spaces, Rendiconti Del Circolo Mathematico Di Palermo (in press).

[5] Hassan Azadi Kenary and Yeol Je Cho, Stability of mixed additivequadratic Jensen type functional equation in various spaces, Computer and Mathematics with Applications, (2011), doi:10.1016/j.camwa.2011.03.024.

[6] P. W. Cholewa, Remarks on the stability of functional equations, Aequationes Mathematicae, 27(1984)76-86. 
[7] S. Czerwik, Functional Equations and Inequalities in Several Variables, World Scientific, River Edge, NJ, 2002.

[8] Z. Gajda, On stability of additive mappings, Internat. J. Math. Math. Sci., 14 (1991), 431-434.

[9] P. Găvruta, A generalization of the Hyers-Ulam-Rassias stability of approximately additive mappings, J. Math. Anal. Appl. 184 (1994), no. 3, 431-436.

[10] D. H. Hyers, On the stability of the linear functional equation, Proc. Nat. Acad. Sci. U.S.A. 27 (1941), 222-224.

[11] D. H. Hyers, G. Isac, and Th. M. Rassias, Stability of Functional Equations in Several Variables, Birkhauser, Basel, 1998.

[12] D. Mihet and V. Radu, On the stability of the additive Cauchy functional equation in random normed spaces, Journal of Math. Anal. and Appl., 343(2008)567-572.

[13] Alireza Kamel Mirmostafaee, Approximately Additive Mappings in NonArchimedean Normed Spaces, Bull. Korean Math. Soc. 46(2009), No.2, pp. 387-400.

[14] C. Park, Fuzzy stability of a functional equation associated with inner product spaces, Fuzzy Sets and Systems 160(2009), 1632-1642.

[15] C. Park, Generalized Hyers-Ulam-Rassias stability of $n$-sesquilinearquadratic mappings on Banach modules over $C^{*}$-algebras, J. Comput. Appl. Math. 180 (2005), 279-291.

[16] C. Park, J. Hou, and S. Oh, Homomorphisms between JC-algebras and Lie $C^{*}$-algebras, Acta Math. Sin. (Engl. Ser.) 21 (2005), no. 6, 1391-1398.

[17] C. Park, On the stability of the quadratic mapping in Banach modules, J. Math. Anal. Appl., 276 (2002), 135-144.

[18] Th. M. Rassias, On the stability of the linear mapping in Banach spaces, Proc. Amer. Math. Soc. 72 (1978), no. 2, 297-300.

[19] Th. M. Rassias, Problem 16; 2, Report of the 27th International Symposium Functional Equations, Aequationes Math., 39 (1990), 292-309. 
[20] Th. M. Rassias, P. Šemrl, On the behaviour of mappings which do not satisfy Hyers-Ulam stability, Proc. Amer. Math. Soc., 114 (1992), 989-993.

[21] R. Saadati, M. Vaezpour and Y.J. Cho, A note to paper "On the stability of cubic mappings and quartic mappings in random normed spaces", J. Ineq. Appl., Volume 2009, Article ID 214530, doi: 10.1155/2009/214530.

[22] R. Saadati and C. Park, Non-Archimedean $\mathcal{L}$-fuzzy normed spaces and stability of functional equations (in press).

[23] R. Saadati, M. Vaezpour and Y. J. Cho, A note to paper "On the stability of cubic mappings and quartic mappings in random normed spaces", J. Ineq. Appl., Volume 2009, Article ID 214530, doi: 10.1155/2009/214530.

[24] Reza Saadati, M. M. Zohdi, and S. M. Vaezpour, Nonlinear L-Random Stability of an ACQ Functional Equation, Journal of Inequalities and Applications, Volume 2011, Article ID 194394, 23 pages, doi:10.1155/2011/194394.

[25] F. Skof, Local properties and approximation of operators, Rend. Sem. Mat. Fis. Milano 53 (1983), 113-129.

[26] S. M. Ulam, Problems in Modern Mathematics, Science Editions, John Wiley and Sons, 1964. 